\title{
Criterion-related validity and reliability of the Urdu version of the patient health questionnaire in community-based pregnant women in Pakistan
}

\author{
John A Gallis ${ }^{1,2}$, Joanna Maselko ${ }^{3}$, Karen O'Donnell ${ }^{2,4}$ ， Ke Song ${ }^{1,2}$, Kiran Saqib ${ }^{5}$, Elizabeth L Turner ${ }^{1,2}$, \\ Siham Sikander Corresp. 5, 6 \\ 1 Department of Biostatistics and Bioinformatics, Duke University, Durham, North Carolina, United States \\ 2 Duke Global Health Institute, Duke University, Durham, North Carolina, United States \\ 3 Department of Epidemiology, Gillings School of Public Health, University of North Carolina at Chapel Hill, Chapel Hill, North Carolina, United States \\ 4 Center for Child and Family Health, Durham, North Carolina, United States \\ 5 Human Development Research Foundation, Islamabad, Pakistan \\ 6 Health Services Academy, Islamabad, Pakistan \\ Corresponding Author: Siham Sikander \\ Email address: siham.sikander@hsa.edu.pk
}

Background. Depression is one of the most prevalent, yet unrecognized but treatable mental disorders in low and middle income countries (LMICS). In such locations, screening tools that are easy-toadminister, valid, and reliable are needed to assist in detecting symptoms of depression. The Patient Health Questionnaire (PHQ-9) is one of the most widely used depression screeners. However, its applicability to community-based settings of Pakistan is limited by the lack of studies examining its validity and reliability in such settings. The current study aimed to demonstrate the criterion-related validity and internal reliability of the Urdu version of the PHQ-9 in community-based pregnant women in Pakistan compared to a diagnostic clinical interview, the Structured Clinical Interview for DSM disorders (SCID), using data from a depression treatment cluster randomized trial in rural Pakistan.

Methods. Pregnant women in a rural, low income sub-district in Pakistan were approached between October 2014 to February 2016 and, after providing informed consent, screened for depression using the Urdu version of the PHQ-9, with a cutoff of $\geq 10$ used to indicate significant depressive symptoms. Following the PHQ-9, the diagnostic module for current major depressive episode of the SCID was administered. We examined the psychometric properties of PHQ-9 compared to SCID as a gold standard, using sensitivity, specificity, and negative and positive predictive value to measure the criterion-related validity of the PHQ-9 as an indicator of symptoms of depression. We computed area under the receiver operating characteristic curve to determine diagnostic accuracy, and used Cronbach's alpha to assess internal reliability.

Results. A total of 1731 women in their third trimester of pregnancy were assessed for major depressive disorder. Of these women, 572 (33\%) met the cutoff for significant depressive symptoms on PHQ-9, and 454 (26\%) were assessed positive for depression using the SCID. The sensitivity and specificity of PHQ-9 at a cutoff of $\geq 10$ was $94.7 \%$ and $88.9 \%$, respectively. The positive and negative predictive values were $75.2 \%$ and $97.9 \%$, respectively; and the area under the curve was 0.959 . Internal reliability, as measured by Cronbach's alpha, was 0.844 .

Discussion. Valid and reliable screening tools to assist in detecting symptoms of depressive disorder are needed in low income settings where depressive disorders are highly prevalent. The Urdu version of the PHQ-9 has not been previously validated against a well-known assessment of depression in a community 
setting among pregnant women in Pakistan. This study demonstrates that the Urdu version of the PHQ-9 has acceptable criterion-related validity and reliability for screening for depressive symptoms in Pakistan among community-based pregnant women; and when the recommended cut-off score of $\geq 10$ is used it can also serve as an accurate screening tool for major depressive disorder. 
1 Criterion-related validity and reliability of the Urdu version of the patient health

2 questionnaire in community-based pregnant women in Pakistan

3 John A. Gallis ${ }^{1,2}$, Joanna Maselko ${ }^{3}$, Karen O’Donnell ${ }^{2,4}$, Ke Song ${ }^{1,2}$, Kiran Saqib ${ }^{5}$, Elizabeth L.

4 Turner ${ }^{1,2}$, Siham Sikander ${ }^{5,6}$

$5{ }^{1}$ Duke Global Health Institute, Duke University, Durham, North Carolina, USA

6 2Department of Biostatistics and Bioinformatics, Duke University, Durham, North Carolina, 7 USA

8 32Department of Epidemiology, Gillings School of Public Health, UNC, Chapel Hill, North

9 Carolina, USA

$10{ }^{4}$ Center for Child and Family Health, Duke University, Durham, North Carolina, USA

$11{ }^{5}$ Human Development Research Foundation, Islamabad, Pakistan

$12{ }^{6}$ Health Services Academy, Islamabad, Pakistan

15 Corresponding Author:

16 Siham Sikander

17

18 Email Address: siham.sikander@hsa.edu.pk 


\section{Abstract}

Background. Depression is one of the most prevalent, yet unrecognized but treatable mental disorders in low and middle income countries (LMICs). In such locations, screening tools that are easy-to-administer, valid, and reliable are needed to assist in detecting symptoms of depression. The Patient Health Questionnaire (PHQ-9) is one of the most widely used depression screeners. However, its applicability to community-based settings of Pakistan is limited by the lack of studies examining its validity and reliability in such settings. The current study aimed to demonstrate the criterion-related validity and internal reliability of the Urdu version of the PHQ-9 in community-based pregnant women in Pakistan compared to a diagnostic clinical interview, the Structured Clinical Interview for DSM disorders (SCID), using data from a depression treatment cluster randomized trial in rural Pakistan.

Methods. Pregnant women in a rural, low income sub-district in Pakistan were approached between October 2014 to February 2016 and, after providing informed consent, screened for depression using the Urdu version of the PHQ-9, with a cutoff of $\geq 10$ used to indicate significant depressive symptoms. Following the PHQ-9, the diagnostic module for current major depressive episode of the SCID was administered. We examined the psychometric properties of PHQ-9 compared to SCID as a gold standard, using sensitivity, specificity, and negative and positive predictive value to measure the criterion-related validity of the PHQ-9 as an indicator of symptoms of depression. We computed area under the receiver operating characteristic curve to determine diagnostic accuracy, and used Cronbach's alpha to assess internal reliability.

Results. A total of 1731 women in their third trimester of pregnancy were assessed for major depressive disorder. Of these women, $572(33 \%)$ met the cutoff for significant depressive symptoms on PHQ-9, and 454 (26\%) were assessed positive for depression using the SCID. The 
42 sensitivity and specificity of PHQ-9 at a cutoff of $\geq 10$ was $94.7 \%$ and $88.9 \%$, respectively. The

43 positive and negative predictive values were $75.2 \%$ and $97.9 \%$, respectively; and the area under

44 the curve was 0.959 . Internal reliability, as measured by Cronbach's alpha, was 0.844 .

45 Discussion. Valid and reliable screening tools to assist in detecting symptoms of depressive

46 disorder are needed in low income settings where depressive disorders are highly prevalent. The

47 Urdu version of the PHQ-9 has not been previously validated against a well-known assessment of

48 depression in a community setting among pregnant women in Pakistan. This study demonstrates

49 that the Urdu version of the PHQ-9 has acceptable criterion-related validity and reliability for

50 screening for depressive symptoms in Pakistan among community-based pregnant women; and

51 when the recommended cut-off score of $\geq 10$ is used it can also serve as an accurate screening tool

52 for major depressive disorder.

53

54 


\section{Introduction}

\section{Scientific and clinical background}

Depressive disorder is a public health priority: It was estimated to be the $15^{\text {th }}$ leading cause of global disability adjusted life years (DALYs) as of 2015, an increase from the $19^{\text {th }}$ leading cause in 1990. (Kassebaum et al. 2016). Depressive disorder was also the second leading cause of years lived with disability (YLDs) in 2010, accounting for an estimated $8.2 \%$ of all YLDs (Ferrari et al. 2013). Depression is one of the most prevalent, yet unrecognized but treatable mental disorders in low and middle income countries (LMICs); easy to administer, valid and reliable screening tools that can assist in detecting depression will be useful in both clinical care and research in geographic areas with limited access to psychiatric professionals.

One of the most widely used screening tools for depressive disorder is the Patient Health Questionnaire (PHQ-9) (Manea et al. 2015). The PHQ-9 was originally developed by Spitzer and colleagues in the mid-1990s, as part of the Primary Care Evaluation of Mental Disorders (PRIME-MD), a diagnostic tool containing modules on 12 different mental health disorders. The PHQ-9 is a tool specific for screening for depressive symptoms, within the mood module of the original PRIME-MD (Kroenke et al. 2001; Spitzer et al. 1999; Spitzer et al. 1994).

The PHQ-9 is based on the Diagnostic and Statistical Manual of Mental Disorders (DSM-IV) criteria of depressive disorder and has nine items with a score ranging from 0 to 3 for each item $(0=$ not at all, $1=$ several days, $2=$ more than half days, $3=$ almost every day of a two-week recall period). The total score ranges from 0-27. Using standard cutoffs, a score of 5 to 9 indicates mild severity of major depression, 10 to 14 indicates moderate severity, 15 to 19 indicates moderately severe depression, while 20 or higher indicates severe major depression (Kroenke et 
77 al. 2001). The 10 point cut-off is most commonly used to indicate symptoms having reached

78 clinically significant levels requiring full diagnostic assessment (Manea et al. 2012). For this study, we generate a continuous total score as well as grade the depressive symptoms using a range of cutoffs.

Over the years, the PHQ-9 has been validated in many settings and has shown to have good sensitivity and specificity when compared to diagnostic interviews (Manea et al. 2015; Moriarty et al. 2015). The PHQ-9 has also been translated into myriad languages, with many translations validated (Adewuya et al. 2006; Bian et al. 2011; Hanwella et al. 2014; Huang et al. 2006; Lotrakul et al. 2008; Wulsin et al. 2002). A number of recent studies on its validity and internal reliability, for screening for depression, and in monitoring treatment response have been published (Chen et al. 2010; Lamers et al. 2008; Monahan et al. 2009; Titov et al. 2011; Wang et al. 2014). As noted above, a score of $\geq 10$ on PHQ-9 has been recommended for use as a diagnostic cut-off for possible major depressive disorder (Kroenke et al. 2001), although more recently researchers have recommended less strict adherence to any single cut-off (Manea et al. 2012). A recent meta-analysis of 36 studies conducted in a range of settings but mainly in highincome countries found that the PHQ-9 cut-off of 10 had 78\% (95\% confidence interval [CI]: $70 \%, 84 \%)$ sensitivity and $87 \%$ (95\% CI: $84 \%, 90 \%)$ specificity for detecting major depressive disorder in primary care and hospital settings (Moriarty et al. 2015), when compared against a "gold standard" such as the structured clinical interview for DSM disorders (SCID).

In Pakistan, the Urdu version PHQ-9 has been used in hospital and community settings as a screening tool for depressive disorder (Fraz et al. 2013; Gholizadeh et al. 2017). Although criterion-related validity of an Urdu version of the PHQ-9 has been tested against the validated Urdu version Self Reporting Questionnaire (SRQ) among patients with coronary artery disease in 
100 an urban setting in Pakistan (Gholizadeh et al. 2017), the PHQ-9 has not been tested for

101 criterion-related validity in a rural setting nor among pregnant or perinatal (that is, including a

102 number of weeks after birth) women in Pakistan. Pregnancy or the entire perinatal period is an

103 especially vulnerable period for women to develop mood disorders, and Pakistan has a very high

104 burden of perinatal depression (Fisher et al. 2012; Rahman et al. 2003a; Saeed et al. 2016).

105 Therefore, having a valid screening tool is useful not only to assist in case identification but also, 106 if compared to a diagnostic criterion tool, for impact evaluation of psychosocial interventions in 107 this population.

108 There is debate around whether and when to screen adults for depression in primary care settings

109 (Thombs et al. 2012; Thombs \& Ziegelstein 2014). The United States Preventive Services task

110 force recommends screening only when adequate resources exist to manage the condition, while

111 the UK and Canadian task forces recommend against it (Thombs \& Ziegelstein 2014). There is

112 even less information on the appropriateness of screening in perinatal populations (Thombs et

113 al. 2014). However, much of this research has focused on high income countries. In rural areas

114 of LMICs where mental health services are virtually non-existent (such as in rural Pakistan), no

115 clear recommendations exist. Benefits and drawbacks of screening for depressive disorder must

116 be weighed considering the health resources that do exist and the negative consequences

117 associated with leaving populations undiagnosed in which the prevalence of depression is

118 estimated to be about 25\% (Rahman et al. 2003a; Saeed et al. 2016).

119 The current study aimed at demonstrating the criterion-related validity and internal reliability of 120 the Urdu version of the PHQ-9 in community-based pregnant women in Pakistan compared to a 121 diagnostic clinical interview, the Structured Clinical Interview for DSM disorders (SCID)

122 (Spitzer et al. 1992). The major depressive episode section of the SCID has previously been 
123 translated into Urdu and culturally adapted (Rahman et al. 2003b), and has been extensively used

124 cross-culturally in studies of depression among pregnant women (Gorman et al. 2004; Nast et al.

125 2013). The primary goal of this study is to determine if the Urdu version of the PHQ-9 provides

126 an adequate tool for testing for depressive symptoms among pregnant women in rural settings in

127 Pakistan by examining its internal reliability and its criterion-related validity relative to the

128 SCID. Ethical approval for this study was obtained for this study from both U.S.-based (Duke

129 University) and Pakistan-based (Human Development Research Foundation) institutional review

130 boards (IRBs). In reporting the results of this study, we followed the Standards for Reporting of

131 Diagnostic Accuracy Studies (STARD) 2015 guidelines (Cohen et al. 2016).

132 Materials \& Methods

133 Study Design and Setting

134 The current study is part of the Thinking Healthy Programme Peer-Delivered (THPP) and

135 Thinking Healthy Programme Peer-Delivered Plus (THPP+) cluster randomized trials (Duke

136 University IRB approval \#Pro00047609; Human Development Research Foundation IRB

137 approval \#IRB/2014/002 and \#IRB/003/2016) (Sikander et al. 2015; Turner et al. 2016). The

138 trials were conducted in the sub-district Kallar Syedan, Rawalpindi District, Pakistan. The sub-

139 district has a population of about 200,000, and is representative of a typical low-socioeconomic

140 rural area of Potohar Plateau situated in the north of the Punjab Province of Pakistan. Most

141 families depend on subsistence farming, supported by earnings of one or more adult male

142 members serving in the armed forces, or working as government employees, semi-skilled, or

143 unskilled laborers in the nearby cities. The spoken and written language in the study area is

144 Urdu. Male and female literacy rates are approximately $64 \%$ and $40 \%$, respectively, in rural

145 Punjab province according to the report from the most recent Demographics and Health Survey 
146 (National Institute of Population Studies - NIPS/Pakistan \& ICF International 2013). Mental

147 health services are virtually non-existent in the sub-district.

148 The aim of these trials is to test the effect on perinatal depression of an extended and peer-

149 delivered version of a previously successful intervention (Rahman et al. 2008). All pregnant

150 women, registered with community health workers (called Lady Health Workers) of the sub-

151 district Kallar Syedan, Rawalpindi District, Pakistan, were approached by study personnel and,

152 after providing written (or witnessed, if illiterate) informed consent, screened for depression

153 using the Urdu version of the PHQ-9. Following the PHQ-9, the diagnostic module for current

154 major depressive episode of the Structured Clinical Interview for DSM disorders (SCID) was

155 administered by the same assessor within the same interview (Spitzer et al. 1992).

156 The study was conducted from October 2014 to February 2016, coinciding with screening and

157 baseline data collection for the THPP and THPP+ trials (Sikander et al. 2015; Turner et al.

158 2016). All interviews were administered by study personnel at the households of the participants

159 or at the house of the Lady Health Worker, whichever was more convenient for the pregnant

160 women. The details of the trials and the recruitment criteria of its community-based depressed

161 and non-depressed pregnant women can be found in the respective protocol papers (Sikander et

162 al. 2015; Turner et al. 2016). In brief, to be eligible for screening, women must be married, in

163 their third trimester of pregnancy, 18 years of age or older, and intend to remain in the study area

164 for at least 1 year. Women are ineligible if they do not speak one of the study languages (Urdu,

165 Punjabi or Potohari) or if they require immediate inpatient care for any reason. Target sample

166 size for the THPP + trial was determined based on the estimated effect size of the primary

167 outcome between intervention and control arms at 36 months (Turner et al. 2016). 
168 In total, 1910 pregnant women across the sub-district were approached for consent and screening

169 for depressive symptoms; 25 (1.3\%) women refused screening while 154 (8.1\%) were ineligible

170 for enrollment. Of those ineligible, $110(71.4 \%)$ were women in their $1^{\text {st }}$ or $2^{\text {nd }}$ trimester of

171 pregnancy, $8(5.2 \%)$ were younger than 18 years old and not able to provide consent, $11(7.1 \%)$

172 did not intend to remain in the study area, $22(14.3 \%)$ did not speak one of the study languages,

173 and $3(1.9 \%)$ required immediate inpatient care. Thus, 1731 community-based pregnant women

174 in their $3^{\text {rd }}$ trimester of pregnancy were screened for current major depressive episode using the

175 Urdu version PHQ-9, which was followed by the SCID diagnostic tool during the same

176 interview. Of these, 1154 were retained for the baseline sample of THPP + because of the

177 sampling design, for which the goal was to recruit into the study every woman who was

178 considered depressed (PHQ-9 $\geq 10$ ), and approximately 1 out of every 3 non-depressed (PHQ-9

$179<10$ ) women screened, based on the estimated prevalence of depression in the population

180 (Sikander et al. 2015; Turner et al. 2016). Criterion-related validity and internal reliability of the

181 PHQ-9 were estimated in the full screening sample $(\mathrm{n}=1731)$. All members of the research team

182 conducting the interviews had a master's degree in either psychology or behavioral sciences, and

183 all of the team members had extensive experience of administering diagnostic interviews for

184 depression within the same study area (Maselko et al. 2015; Turner et al. 2016). They were also

185 supervised weekly by a trained and experienced psychiatrist.

186 Test Methods

187 The translated Urdu version of the PHQ-9 was used as the index test (Multicultural Mental

188 Health Resource Centre). Prior to its use, some difficult Urdu words were replaced by simpler

189 and more frequently used words carrying the same meaning. This was done to help address any

190 potential issue of comprehensibility when administered in a low literacy rural population. For 
191 example, a difficult and not commonly used Urdu word for "feeling down" was changed to an

192 easier Urdu word with the same meaning. This was done by a panel of mental health experts with

193 extensive clinical and field experience of working in low literacy populations. Comprehensibility

194 was examined by pilot testing the PHQ-9 among 250 pregnant women prior to the start to the

195 study. These women were also asked if any of the items were not understood. None of these

196250 women were part of the trial screening sample, and no adjustments were made to the index

197 test as a result of the pilot testing.

198

199

The translated Urdu version of the major depressive episode section of the SCID was used as the reference standard. The SCID was chosen as the reference standard because the Urdu version of this tool was previously translated and culturally adapted for a prior study in this region using a rigorous procedure (Rahman et al. 2003b; Rahman et al. 2008). The SCID is a semi-structured interview that generates case vs non-case diagnosis of current major depressive episode by inquiring about the individual symptoms of depression. To make a diagnosis of depression at least 5 symptoms are needed, including depressed mood or loss of interest (Spitzer et al. 1992).

The Urdu and English version of both the index and reference test are included as a supplementary file.

As previously mentioned, a cutoff of 10 or above on the PHQ-9 was used to identify women with sufficient depressive symptoms to indicate a high probability of a depressive disorder. This is a commonly used cutoff (Manea et al. 2015; Moriarty et al. 2015), and was chosen for the THPP

212 and THPP + trials because of its high positive predictive value for the diagnosis of depressive disorder in the (non-Pakistan) literature (Sikander et al. 2015). Evaluating the validity of the 
214 cutoff of 10 or above on the PHQ-9 was prespecified based on the trial design. We also

215 examined the sensitivity and specificity of cutoffs ranging from 5 to 20 as an exploratory

216 analysis. Since the same interviewer administered both the PHQ-9 and SCID, the assessors were

217 not blinded to the results of the PHQ-9 when administering the SCID.

218 Statistical Analysis

219 In order to test the criterion-related validity and reliability of scores from the Urdu version of

220 PHQ-9, we examined the psychometric properties of PHQ-9 using SCID as a gold standard,

221 using the THPP and THPP+ screening dataset of 1731 women. There was no missing data, as all

222 items from the PHQ-9 and a diagnosis from the SCID were available on all women screened, and

223 no index test or reference standard results were indeterminate. To verify the association between

224 PHQ-9 and SCID, we assessed the criterion-related validity of PHQ-9 in Urdu in reference to the

225 SCID by calculating sensitivity, specificity, positive predictive value (PPV), and negative

226 predictive value (NPV) using cutoff values ranging from 5 to 20. Sensitivity and specificity of

227 cutoffs were also visualized using a receiver operating characteristic (ROC) curve, from which

228 was computed area under the curve (AUC). The internal reliability of the Urdu version of the

229 PHQ-9 was assessed based on the value of the overall Cronbach's alpha. We also computed the

230 Cronbach's alpha with each item deleted to further examine the inter-item consistency. Data

231 were analyzed using Stata 15 (StataCorp, College Station TX, USA).

\section{Results}

233 Participants

234 A total of 1731 women were screened for inclusion in the study. Overall, women had a mean

235 (standard deviation [SD]) PHQ-9 score of 6.8 (6.3) [range: 0-27] in the screening sample. Table 
2361 displays demographic information that was additionally available on the 1,154 women who

237 were invited to participate in the study based on the study design protocol requirements (Turner

238 et al. 2016). These women had a mean (SD) PHQ-9 score of 8.7 (6.7) [range: 0-27] and mean

239 (SD) age of 26.7 (4.5) years. To utilize the most data, we only report validity and reliability

240 results for the full screened sample ( $\mathrm{n}=1731)$. If we used 10 or above as our cutoff for PHQ-9,

241 approximately one-third of the women screened were considered to be at least moderately

242 depressive. However, there were relatively fewer women (26\%) who were diagnosed with

243 depression based upon the results of SCID. The distribution of PHQ-9 scores and SCID results

244 among all women screened are shown in Table 2. In comparison with SCID, the moderate

245 severity group (scoring from 10 to 14 ) had the largest inconsistency, since $112(8.8 \%)$ women

246 who were not depressed according to SCID had moderate depression severity according to PHQ-

2479 results.

248 Test Results

249 Criterion-related validity test results are reported in Table 3 . The sensitivity and specificity of 250 PHQ-9 for a cutoff of 10 or more were $94.7 \%(95 \%$ CI $92.2 \%, 96.6 \%)$ and $88.9 \%(95 \%$ CI $87 \%$, 251 90.6\%), respectively. The PPV and NPV at this cutoff were 75.2\% (95\% CI 71.4\%, 78.7\%) and $25297.9 \%(95 \%$ CI 96.9\%, 98.7\%). The tradeoff between sensitivity and specificity at each cutoff is

253 also shown in Figure 1. The area under the curve (AUC) was 0.959 (95\% CI 0.950, 0.968).

254 According to the results in Table 3 and Figure 1, a cutoff of 10 or more seemed to be a good choice 255 for using PHQ-9 as a depression screener, given high sensitivity and specificity. However, any 256 cutoff between 9 or more and 11 or more appeared to have good sensitivity and specificity, with 257 the estimated sensitivity and specificity values above $85 \%$. In their systematic review, Manea et 
258 al. (2012) also found cutoffs in this range similar to the cutoff of 10 or more based on psychometric 259 properties.

260 We assessed internal reliability based on the overall standardized Cronbach's alpha for all items

261 together and alpha when each item is deleted. The overall standardized Cronbach's alpha was

2620.844 , indicating a high internal consistency. The alpha if item deleted ranged from 0.809 to 0.842 ,

263 which suggested that internal consistency remained unaffected even if one of the items was

264 removed. Results are shown in Table 4.

\section{Discussion}

266

267

268

269

270

271

272

273

274

275

Depressive disorders are highly prevalent and issues around screening and accurate diagnosis are still being debated (Manea et al. 2012), with some countries recommending screening only under certain circumstances, and other countries recommending against routine screening in primary care (Thombs et al. 2012; Thombs \& Ziegelstein 2014). In rural areas of LMICs where mental health services are limited, the benefits and drawbacks of screening for depressive disorder have to be weighed considering the health resources that do exist and the dangers associated with leaving populations undiagnosed. Valid and reliable screening tools to assist in detecting depressive disorder are much needed in low income settings where depressive disorders are more prevalent, such as in Pakistan, and where access to full diagnostic work-up is extremely limited. These valid and reliable screening tools are especially needed as more low-cost, effective community-based interventions are implemented and scaled up in these rural areas (Rahman et al. 2008). While Urdu versions of several instruments for detecting depressive disorders have been validated in Pakistani settings, the Urdu version of the PHQ-9 has not been tested for criterion-related validity in a community setting in Pakistan (Ahmer et al. 2007; Gholizadeh et al. 2017). 
281 Through piloting the PHQ-9 in Urdu, we found that it was easily understood by community-based

282 pregnant women, who answered both somatic and psychological items with equal ease. Using a

283 diagnostic interview for comparison, data from the full screening sample of 1731 pregnant women

284 showed the Urdu version of the PHQ-9 had good criterion-related validity, similar to that reported

285 in other studies (Bian et al. 2011; Williams et al. 2005). The Urdu version PHQ-9 also had a high

286 internal reliability $($ Cronbach's alpha $=0.844)$ in this sample. The internal reliability in the current

287 study is comparable to what other studies report within varied populations with alpha value of

288 0.85-0.90 (Adewuya et al. 2006; Hanwella et al. 2014; Martin et al. 2006).

We note that for a cutoff of $\geq 10$ the specificity is right in line with the pooled (from 36 studies)

specificity obtained in Moriarty et al. (2015), however, the sensitivity is well above the upper limit of the pooled $95 \%$ confidence interval reported. This could be because of the interviewer administered both the SCID and PHQ-9 in the current study or because the studies summarized in Moriarty et al. (2015) do not include populations culturally similar to Pakistan. Also, the studies reported in Moriarty et al. (2015) included mainly hospital or clinic samples, rather than a community-based population. The high value of the AUC (0.959) in this study demonstrates diagnostic accuracy which is in line with a number of studies (Hanwella et al. 2014; Kroenke et al. 2001; Martin et al. 2006).

299

The most reliable estimates (using a clinical interview rather than a screener) among antenatal women in rural Pakistan show a depression prevalence of 23-26\% (Rahman et al. 2004; Rahman et al. 2008), in line with the estimate from our study based on SCID (26\%). Linking results from 
304 specificity of $94.7 \%$ and $88.9 \%$, respectively) in a population where $26 \%$ of pregnant women are

305 depressed corresponds to approximately 3 out of $4(75.2 \%)$ new cases identified by the PHQ-9

306 being true cases.

307

308 One of the limitations of our study was that the same research team administered PHQ-9 and SCID 309 in a single phase design and were not blind to PHQ-9 scores of the study participants when they 310 administered the SCID. This is a potential source of interviewer bias, since knowing the results of 311 one test may influence the interviewer's assessment of the woman on the other test. However, the 312 interviewers were not aware that the data being collected would contribute to a validation study.

313 In addition, each interviewer administered the SCID to a wide range of PHQ-9 high and low 314 scoring participants, thus minimizing any potential interviewer bias of not being blind to PHQ-9 315 scores.

Another limitation of and potential source of bias in this study is that these women were not asked 318 for previous medical records or diagnosis of depression they may have had. Including women with a prior diagnosis of depressive disorder in our study could lead to a sample with increased severity and prevalence of depression, which may inflate the reported sensitivity of PHQ-9 in relation to the SCID (Thombs et al. 2011). However, in this rural Pakistan setting medical histories/records are generally not kept or maintained and if available are not considered reliable. Additionally, given the lack of mental health services in this area, the probability of the women in this study area having had a previous diagnosis of depression is vanishingly small. Finally, the percentage of women $(26 \%)$ in the screened sample who were diagnosed with depression based upon the results of the SCID is in line with the reported prevalence of depressive disorder during 
327 pregnancy of $23-26 \%$ from studies conducted in the same area of rural Pakistan (Rahman et al.

328 2004; Rahman et al. 2008).

\section{Conclusions}

330 This study demonstrates that the Urdu version of the PHQ-9 has acceptable criterion-related

331 validity and reliability for screening for depressive symptoms in Pakistan among community-based

332 pregnant women; and when the recommended cut-off score of $\geq 10$ is used it can also serve as an 333 accurate screening tool for major depressive disorder.

\section{Acknowledgements}

336 We would like to acknowledge the team members working on these projects and for their

337 contributions, namely, Tayyiba Abbasi, Ikhlaq Ahmad, Qurat-ul-Ain, Najia Atif, Amina Bibi,

338 Samina Bilal, Sonia Khan, Rakshanda Liaqat, Anum Nisar, Atif Rahman, Maria Sharif, Shaffaq

339 Zufiqar, Ahmed Zaidi. We would also like to thank the editor and two reviewers for providing

340 helpful comments which improved the final version of this manuscript.

341

342

343 


\section{References}

Adewuya AO, Ola BA, and Afolabi 0O. 2006. Validity of the patient health questionnaire (PHQ-9) as a screening tool for depression amongst Nigerian university students. Journal of Affective Disorders 96:89-93.

Ahmer S, Faruqui RA, and Aijaz A. 2007. Psychiatric rating scales in Urdu: a systematic review. BMC Psychiatry 7:59.

Bian C, Li C, Duan Q, and Wu H. 2011. Reliability and validity of patient health questionnaire: depressive syndrome module for outpatients. Scientific Research and Essays 6:278-282.

Chen S, Chiu H, Xu B, Ma Y, Jin T, Wu M, and Conwell Y. 2010. Reliability and validity of the PHQ-9 for screening late-life depression in Chinese primary care. International Journal of Geriatric Psychiatry 25:1127-1133.

Cohen JF, Korevaar DA, Altman DG, Bruns DE, Gatsonis CA, Hooft L, Irwig L, Levine D, Reitsma JB, and De Vet HC. 2016. STARD 2015 guidelines for reporting diagnostic accuracy studies: explanation and elaboration. BMJ open 6:e012799.

Ferrari AJ, Charlson FJ, Norman RE, Patten SB, Freedman G, Murray CJL, Vos T, and Whiteford HA. 2013. Burden of Depressive Disorders by Country, Sex, Age, and Year: Findings from the Global Burden of Disease Study 2010. PLoS Medicine 10:e1001547. 10.1371/journal.pmed.1001547

Fisher J, de Mello MC, Patel V, Rahman A, Tran T, Holton S, and Holmes W. 2012. Prevalence and determinants of common perinatal mental disorders in women in low- and lower-middleincome countries: a systematic review. Bulletin of the World Health Organization 90:139-149. 10.2471/blt.11.091850

Fraz K, Khan S, and Sikander S. 2013. Screening for depression in coronary artery disease patients using PHQ-9. Health 4:3-6.

Gholizadeh L, Ali khan S, Vahedi F, and Davidson PM. 2017. Sensitivity and specificity of Urdu version of the PHQ-9 to screen depression in patients with coronary artery disease. Contemporary Nurse 53:75-81.

Gorman LL, O'Hara MW, Figueiredo B, Hayes S, Jacquemain F, Kammerer MH, Klier CM, Rosi S, Seneviratne G, and Sutter-Dallay A-L. 2004. Adaptation of the Structured Clinical Interview for DSM-IV Disorders for assessing depression in women during pregnancy and post-partum across countries and cultures. The British Journal of Psychiatry 184:s17-s23.

Hanwella R, Ekanayake S, and de Silva VA. 2014. The validity and reliability of the Sinhala translation of the Patient Health Questionnaire (PHQ-9) and PHQ-2 Screener. Depression Research and Treatment 2014.

Huang FY, Chung H, Kroenke K, Delucchi KL, and Spitzer RL. 2006. Using the patient health questionnaire-9 to measure depression among racially and ethnically diverse primary care patients. Journal of General Internal Medicine 21:547-552.

Kassebaum NJ, Arora M, Barber RM, Bhutta Z, Carter A, Casey DC, Charlson FJ, Coates MM, Ärnlöv J, and Murray CJ. 2016. Global, regional, and national disability-adjusted life-years (DALYs) for 315 diseases and injuries and healthy life expectancy (HALE), 1990-2015: a systematic analysis for the Global Burden of Disease Study 2015. The Lancet 388:1603-1658.

Kroenke K, Spitzer R, and Williams J. 2001. The PHQ-9: Validity of a brief depression severity measure. . Journal of General Internal Medicine 16:606-613.

Lamers F, Jonkers CC, Bosma H, Penninx BW, Knottnerus JA, and van Eijk JTM. 2008. Summed score of the Patient Health Questionnaire-9 was a reliable and valid method for depression screening in chronically ill elderly patients. Journal of Clinical Epidemiology 61:679-687. 
389

390

391

392

393

394

395

396

397

398

399

400

401

402

403

404

405

406

407

408

409

410

411

412

413

414

415

416

417

418

419

420

421

422

423

424

425

426

427

428

429

430

431

432

433

434

435

Lotrakul M, Sumrithe S, and Saipanish R. 2008. Reliability and validity of the Thai version of the PHQ-9. BMC Psychiatry 8:46.

Manea L, Gilbody S, and McMillan D. 2012. Optimal cut-off score for diagnosing depression with the Patient Health Questionnaire (PHQ-9): a meta-analysis. Canadian Medical Association Journal 184:E191-E196.

Manea L, Gilbody S, and McMillan D. 2015. A diagnostic meta-analysis of the Patient Health Questionnaire-9 (PHQ-9) algorithm scoring method as a screen for depression. General Hospital Psychiatry 37:67-75.

Martin A, Rief W, Klaiberg A, and Braehler E. 2006. Validity of the brief patient health questionnaire mood scale (PHQ-9) in the general population. General Hospital Psychiatry 28:71-77.

Maselko J, Sikander S, Bhalotra S, Bangash O, Ganga N, Mukherjee S, Egger H, Franz L, Bibi A, and Liaqat R. 2015. Effect of an early perinatal depression intervention on long-term child development outcomes: follow-up of the Thinking Healthy Programme randomised controlled trial. The Lancet Psychiatry 2:609-617.

Monahan PO, Shacham E, Reece M, Kroenke K, Ong'or WO, Omollo O, Yebei VN, and Ojwang C. 2009. Validity/reliability of PHQ-9 and PHQ-2 depression scales among adults living with HIV/AIDS in western Kenya. Journal of General Internal Medicine 24:189.

Moriarty AS, Gilbody S, McMillan D, and Manea L. 2015. Screening and case finding for major depressive disorder using the Patient Health Questionnaire (PHQ-9): a meta-analysis. General Hospital Psychiatry 37:567-576. 10.1016/j.genhosppsych.2015.06.012

Multicultural Mental Health Resource Centre. Urdu version of the PHQ-9 and GAD-7. Available at http://www.multiculturalmentalhealth.ca/wp-content/uploads/2013/11/PHQ-9-Urdu.pdf.

Nast I, Bolten M, Meinlschmidt G, and Hellhammer DH. 2013. How to Measure Prenatal Stress? A Systematic Review of Psychometric Instruments to Assess Psychosocial Stress during Pregnancy. Paediatric and Perinatal Epidemiology 27:313-322. 10.1111/ppe.12051

National Institute of Population Studies - NIPS/Pakistan, and ICF International. 2013. Pakistan Demographic and Health Survey 2012-13. Islamabad, Pakistan: NIPS/Pakistan and ICF International.

Rahman A, Iqbal Z, Bunn J, Lovel H, and Harrington R. 2004. Impact of maternal depression on infant nutritional status and illness: A cohort study. Archives of General Psychiatry 61:946-952. 10.1001/archpsyc.61.9.946

Rahman A, Iqbal Z, and Harrington R. 2003a. Life events, social support and depression in childbirth: perspectives from a rural community in the developing world. Psychological Medicine 33:11611167. 10.1017/s0033291703008286

Rahman A, lqbal Z, Waheed W, and Hussain N. 2003b. Translation and cultural adaptation of health questionnaires. The Journal of the Pakistan Medical Association 53:142-147.

Rahman A, Malik A, Sikander S, Roberts C, and Creed F. 2008. Cognitive behaviour therapy-based intervention by community health workers for mothers with depression and their infants in rural Pakistan: a cluster-randomised controlled trial. The Lancet 372:902-909.

Saeed A, Humayun A, and Raana T. 2016. The Alarming Rise in Prevalence of Antenatal Depression in Pakistan: A Review. Proceeding SZPGMI Vol 30:107-111.

Sikander S, Lazarus A, Bangash O, Fuhr DC, Weobong B, Krishna RN, Ahmad I, Weiss HA, Price L, and Rahman A. 2015. The effectiveness and cost-effectiveness of the peer-delivered Thinking Healthy Programme for perinatal depression in Pakistan and India: the SHARE study protocol for randomised controlled trials. Trials 16:1-14.

Spitzer RL, Kroenke K, Williams JB, and Group PHQPCS. 1999. Validation and utility of a self-report version of PRIME-MD: the PHQ primary care study. JAMA 282:1737-1744. 
436

437

438

439

440

441

442

443

444

445

446

447

448

449

450

451

452

453

454

455

456

457

458

459

460

461

462

463

464

465

466

467

Spitzer RL, Williams JB, Gibbon M, and First MB. 1992. The structured clinical interview for DSM-III-R (SCID): I: history, rationale, and description. Archives of General Psychiatry 49:624-629.

Spitzer RL, Williams JB, Kroenke K, Linzer M, Verloin deGruy F, Hahn SR, Brody D, and Johnson JG. 1994. Utility of a new procedure for diagnosing mental disorders in primary care: the PRIME-MD 1000 study. JAMA 272:1749-1756.

Thombs BD, Arthurs E, Coronado-Montoya S, Roseman M, Delisle VC, Leavens A, Levis B, Azoulay L, Smith C, Ciofani L, Coyne JC, Feeley N, Gilbody S, Schinazi J, Stewart DE, and Zelkowitz P. 2014. Depression screening and patient outcomes in pregnancy or postpartum: A systematic review. Journal of Psychosomatic Research 76:433-446. 10.1016/j.jpsychores.2014.01.006

Thombs BD, Arthurs E, El-Baalbaki G, Meijer A, Ziegelstein RC, and Steele RJ. 2011. Risk of bias from inclusion of patients who already have diagnosis of or are undergoing treatment for depression in diagnostic accuracy studies of screening tools for depression: systematic review. BMJ 343. 10.1136/bmj.d4825

Thombs BD, Coyne JC, Cuijpers P, De Jonge P, Gilbody S, loannidis JP, Johnson BT, Patten SB, Turner EH, and Ziegelstein RC. 2012. Rethinking recommendations for screening for depression in primary care. Canadian Medical Association Journal 184:413-418.

Thombs BD, and Ziegelstein RC. 2014. Does depression screening improve depression outcomes in primary care? BMJ : British Medical Journal 348. 10.1136/bmj.g1253

Titov N, Dear BF, McMillan D, Anderson T, Zou J, and Sunderland M. 2011. Psychometric comparison of the PHQ-9 and BDI-II for measuring response during treatment of depression. Cognitive Behaviour Therapy 40:126-136.

Turner EL, Sikander S, Bangash O, Zaidi A, Bates L, Gallis J, Ganga N, O’Donnell K, Rahman A, and Maselko J. 2016. The effectiveness of the peer delivered Thinking Healthy Plus (THPP+) Programme for maternal depression and child socio-emotional development in Pakistan: study protocol for a three-year cluster randomized controlled trial. Trials 17:442.

Wang W, Bian Q, Zhao Y, Li X, Wang W, Du J, Zhang G, Zhou Q, and Zhao M. 2014. Reliability and validity of the Chinese version of the Patient Health Questionnaire (PHQ-9) in the general population. General Hospital Psychiatry 36:539-544.

Williams LS, Brizendine EJ, Plue L, Bakas T, Tu W, Hendrie H, and Kroenke K. 2005. Performance of the PHQ-9 as a screening tool for depression after stroke. Stroke 36:635-638.

Wulsin L, Somoza E, and Heck J. 2002. The feasibility of using the Spanish PHQ-9 to screen for depression in primary care in Honduras. Primary Care Companion to the Journal of Clinical Psychiatry 4:191. 


\section{Table $\mathbf{1}$ (on next page)}

Baseline participant demographic characteristics, by PHQ-9 and SCID, n=1154

Demographic characteristics are from a 1:1 depressed/non-depressed sample (as determined by PHQ-9 $\geq 10$ ) of $n=1154$ women. Note: The baseline sample of $n=1154$ is a subset of the screening sample of $n=1731$. 


\section{Table 1:}

2 Baseline Participant Demographic Characteristics, by PHQ-9 and SCID, $n=1154$

PHQ-9 Cutoff at 10

\begin{tabular}{|c|c|c|c|c|c|}
\hline & $\begin{array}{c}\text { Not Depressed } \\
(\mathrm{N}=584)\end{array}$ & $\begin{array}{c}\text { Depressed } \\
(\mathrm{N}=570)\end{array}$ & $\begin{array}{c}\text { Not Depressed } \\
(\mathrm{N}=712)\end{array}$ & $\begin{array}{c}\text { Depressed } \\
(\mathrm{N}=442)\end{array}$ & $\begin{array}{c}\text { Total } \\
(\mathrm{N}=1154) \\
\end{array}$ \\
\hline \multicolumn{6}{|l|}{ Age (in years) } \\
\hline Mean (SD) & $26.4(4.3)$ & $27.0(4.8)$ & $26.5(4.3)$ & $27.0(4.8)$ & $26.7(4.5)$ \\
\hline Median (Q1, Q3) & $26.0(23.0,29.0)$ & $27.0(24.0,30.0)$ & $26.0(24.0,30.0)$ & $27.0(23.0,30.0)$ & $26.0(23.0,30.0)$ \\
\hline Range & $(18.0-40.0)$ & $(18.0-45.0)$ & $(18.0-40.0)$ & $(18.0-45.0)$ & $(18.0-45.0)$ \\
\hline \multicolumn{6}{|l|}{$\begin{array}{l}\text { Total \# of Children in the } \\
\text { Household }\end{array}$} \\
\hline Mean (SD) & $2.5(2.6)$ & $3.0(2.7)$ & $2.6(2.6)$ & $3.1(2.7)$ & $2.8(2.7)$ \\
\hline Median (Q1, Q3) & $2.0(1.0,4.0)$ & $2.0(1.0,4.0)$ & $2.0(1.0,4.0)$ & $2.0(1.0,4.0)$ & $2.0(1.0,4.0)$ \\
\hline Range & $(0.0-20.0)$ & $(0.0-21.0)$ & $(0.0-20.0)$ & $(0.0-21.0)$ & $(0.0-21.0)$ \\
\hline \multicolumn{6}{|l|}{ PHQ-9 Total Score } \\
\hline Mean (SD) & $2.8(2.5)$ & $14.7(3.7)$ & $4.7(4.7)$ & $15.1(4.0)$ & $8.7(6.7)$ \\
\hline Median (Q1, Q3) & $3.0(0.0,4.0)$ & $14.0(12.0,17.0)$ & $3.0(1.0,7.0)$ & $15.0(12.0,18.0)$ & $9.0(3.0,14.0)$ \\
\hline Range & $(0.0-9.0)$ & $(10.0-27.0)$ & $(0.0-25.0)$ & $(2.0-27.0)$ & $(0.0-27.0)$ \\
\hline \multicolumn{6}{|l|}{$\begin{array}{l}\text { Grades woman } \\
\text { has passed }\end{array}$} \\
\hline None $(0)$ & $63(10.8 \%)$ & $107(18.8 \%)$ & $83(11.7 \%)$ & $87(19.7 \%)$ & $170(14.7 \%)$ \\
\hline Primary (1-5) & $87(14.9 \%)$ & $139(24.4 \%)$ & $124(17.4 \%)$ & $102(23.1 \%)$ & $226(19.6 \%)$ \\
\hline Middle (6-8) & $108(18.5 \%)$ & $107(18.8 \%)$ & $129(18.1 \%)$ & $86(19.5 \%)$ & $215(18.6 \%)$ \\
\hline Secondary (9-10) & $167(28.6 \%)$ & $126(22.1 \%)$ & $191(26.8 \%)$ & $102(23.1 \%)$ & $293(25.4 \%)$ \\
\hline Higher Secondary (11-12) & $63(10.8 \%)$ & $46(8.1 \%)$ & $74(10.4 \%)$ & $35(7.9 \%)$ & $109(9.4 \%)$ \\
\hline Tertiary $(>12)$ & $96(16.4 \%)$ & $45(7.9 \%)$ & $111(15.6 \%)$ & $30(6.8 \%)$ & $141(12.2 \%)$ \\
\hline \multicolumn{6}{|l|}{$\begin{array}{l}\text { Grades husband } \\
\text { has passed }\end{array}$} \\
\hline None $(0)$ & $33(5.7 \%)$ & $55(9.6 \%)$ & $42(5.9 \%)$ & $46(10.4 \%)$ & $88(7.6 \%)$ \\
\hline Primary (1-5) & $45(7.7 \%)$ & $67(11.8 \%)$ & $62(8.7 \%)$ & $50(11.3 \%)$ & $112(9.7 \%)$ \\
\hline Middle (6-8) & $105(18.0 \%)$ & $137(24.0 \%)$ & $132(18.5 \%)$ & $110(24.9 \%)$ & $242(21.0 \%)$ \\
\hline Secondary (9-10) & $286(49.0 \%)$ & $243(42.6 \%)$ & $347(48.7 \%)$ & $182(41.2 \%)$ & $529(45.8 \%)$ \\
\hline Higher Secondary (11-12) & $65(11.1 \%)$ & $47(8.2 \%)$ & $73(10.3 \%)$ & $39(8.8 \%)$ & $112(9.7 \%)$ \\
\hline Tertiary (>12) & $50(8.6 \%)$ & $21(3.7 \%)$ & $56(7.9 \%)$ & $15(3.4 \%)$ & $71(6.2 \%)$ \\
\hline
\end{tabular}

3 Demographic characteristics are from a 1:1 depressed/non-depressed sample (as determined by PHQ-9 $\geq 10$ ) of

$4 n=1154$ women. Note: The baseline sample of $n=1154$ is a subset of the screening sample of $n=1731$. 
Table 2 (on next page)

PHQ-9 Score cutoffs by SCID reference standard; screening sample, $n=1731$ 
1 Table 2:

2 PHQ-9 Score cutoffs by SCID reference standard; screening sample, $n=1731$

\begin{tabular}{l|cc|c}
\hline \multicolumn{3}{|c}{ SCID Depression Status } \\
PHQ-9 Severity of Depression & $\begin{array}{c}\text { Depressed } \\
(\mathrm{N}=454)\end{array}$ & $\begin{array}{c}\text { Non depressed } \\
(\mathrm{N}=1277)\end{array}$ & $\begin{array}{c}\text { Total } \\
(\mathrm{N}=1731)\end{array}$ \\
\hline Minimal, 0-4 & $5(1.1 \%)$ & $850(66.6 \%)$ & $855(49.4 \%)$ \\
Mild, 5-9 & $19(4.2 \%)$ & $285(22.3 \%)$ & $304(17.6 \%)$ \\
Moderate, 10-14 & $202(44.5 \%)$ & $112(8.8 \%)$ & $314(18.1 \%)$ \\
Moderately Severe, 15-19 & $160(35.2 \%)$ & $27(2.1 \%)$ & $187(10.8 \%)$ \\
Severe, 20+ & $68(15.0 \%)$ & $3(0.2 \%)$ & $71(4.1 \%)$ \\
\hline
\end{tabular}

3 


\section{Table 3(on next page)}

Psychometric properties of PHQ-9 at several cutoffs, with SCID as the reference standard; screening sample, $\mathrm{n}=1731$

All numbers are percentages; using data from $n=1731$ women screened for depression. 
Table 3:

Psychometric properties of PHQ-9 at several cutoffs, with SCID as the reference standard; screening sample, $n=1731$

\begin{tabular}{|c|c|c|c|c|}
\hline $\begin{array}{l}\text { PHQ-9 } \\
\text { Cutoffs }\end{array}$ & $\begin{array}{r}\text { Sensitivity } \\
(95 \% \mathrm{Cl})\end{array}$ & $\begin{array}{r}\text { Specificity } \\
(95 \% \mathrm{Cl})\end{array}$ & $\begin{array}{r}\text { Positive } \\
\text { Predictive Value } \\
(95 \% \mathrm{Cl})\end{array}$ & $\begin{array}{r}\text { Negative } \\
\text { Predictive Value } \\
(95 \% \mathrm{Cl})\end{array}$ \\
\hline$\geq 5$ & $98.9(97.4,99.6)$ & $66.6(63.9,69.1)$ & $51.3(47.9$, & $99.4(98$ \\
\hline$\geq 6$ & $98.0(96.3,99.1)$ & $74.1(71.6,76.5)$ & $57.3(53.8,60.9)$ & $99.1(98.2,99.6)$ \\
\hline$\geq 7$ & $97.4(95.4,98.6)$ & $80.9(78.6,83.0)$ & $64.4(60.7,68.0)$ & $98.9(98.0,99.4)$ \\
\hline$\geq 8$ & $96.5(94.3,98.0)$ & $84.9(82.8,86.8)$ & $69.4(65.7,73.0)$ & $98.5(97.6,99.2)$ \\
\hline$\geq 9$ & $95.8(93.5,97.5)$ & $87.9(85.9,89.6)$ & $73.7(70.0,77.2)$ & $98.3(97.4,99.0)$ \\
\hline$\geq 10$ & $94.7(92.2,96.6)$ & $88.9(87.0,90.6)$ & $75.2(71.4,78.7)$ & $97.9(96.9,98.7)$ \\
\hline$\geq 11$ & $89.0(85.7,91.7)$ & $91.9(90.3,93.4)$ & $79.7(75.9,83.1)$ & $95.9(94.6,97.0)$ \\
\hline$\geq 12$ & $81.1(77.1,84.6)$ & $94.0(92.6,95.3)$ & $82.9(79.1,86.3)$ & $93.3(91.8,94.6)$ \\
\hline$\geq 13$ & $70.0(65.6,74.2)$ & $95.5(94.3,96.6)$ & $84.8(80.8,88.3)$ & $90.0(88.2,91.5)$ \\
\hline$\geq 14$ & $59.9(55.2,64.5)$ & $96.6(95.4,97.5)$ & $86.1(81.8,89.7)$ & $87.1(85.3,88.8)$ \\
\hline$\geq 15$ & $50.2(45.5,54.9)$ & $97.7(96.7,98.4)$ & $88.4(83.8,92.0)$ & $84.7(82.7,86.5)$ \\
\hline$\geq 16$ & $39.2(34.7,43.9)$ & $98.3(97.4,98.9)$ & $89.0(83.8,93.0)$ & $82.0(80.0,83.9)$ \\
\hline$\geq 17$ & $33.9(29.6,38.5)$ & $98.7(98.0,99.3)$ & $90.6(85.2,94.5)$ & $80.8(78.7,82.7)$ \\
\hline$\geq 18$ & $26.2(22.2,30.5)$ & $99.2(98.6,99.6)$ & $92.2(86.2,96.2)$ & $79.1(77.0,81.1)$ \\
\hline$\geq 19$ & $19.6(16.0,23.6)$ & $99.5(98.9,99.8)$ & $92.7(85.6,97.0)$ & $77.7(75.6,79.7)$ \\
\hline$\geq 20$ & $15.0(11.8,18.6)$ & $99.8(99.3,100.0)$ & $95.8(88.1,99.1)$ & $76.7(74.6,78.8)$ \\
\hline
\end{tabular}

All numbers are percentages; using data from $n=1731$ women screened for depression. 


\section{Table 4(on next page)}

Cronbach's alpha (internal reliability) results; screening sample, $\mathrm{n}=1731$

*Note: questions were asked in a different order than is standard **Items were standardized 
1 Table 4:

2 Cronbach's alpha (internal reliability) results; screening sample, $n=1731$

\begin{tabular}{l|c}
\hline PHQ-9 Items* & $\begin{array}{c}\text { Cronbach's Alpha } \\
\text { if Item Deleted }\end{array}$ \\
\hline 1. Feeling tired or having little energy & 0.828 \\
2. Poor appetite or overeating & 0.842 \\
3. Trouble falling or staying asleep, or sleeping too much & 0.836 \\
4. Moving or speaking slowly & 0.820 \\
5. Trouble concentrating & 0.836 \\
6. Little interest or pleasure in doing things & 0.816 \\
7. Feeling down, depressed, or hopeless & 0.809 \\
8. Feeling bad about yourself & 0.824 \\
9. Thoughts that you would better off dead, or of hurting yourself & 0.839 \\
\hline Overall Cronbach's Alpha & $\mathbf{0 . 8 4 4}$ \\
\hline
\end{tabular}

*Note: questions were asked in a different order than is standard

$4 * *$ Items were standardized

5 
Figure 1 (on next page)

Receiver operating characteristic (ROC) curve comparing different cutoffs of PHQ-9 score to the SCID reference standard; screening sample, $n=1731$.

Abbreviation: AUC - Area Under the Curve. Bolded point indicates the cutoff of $\geq 10$. 


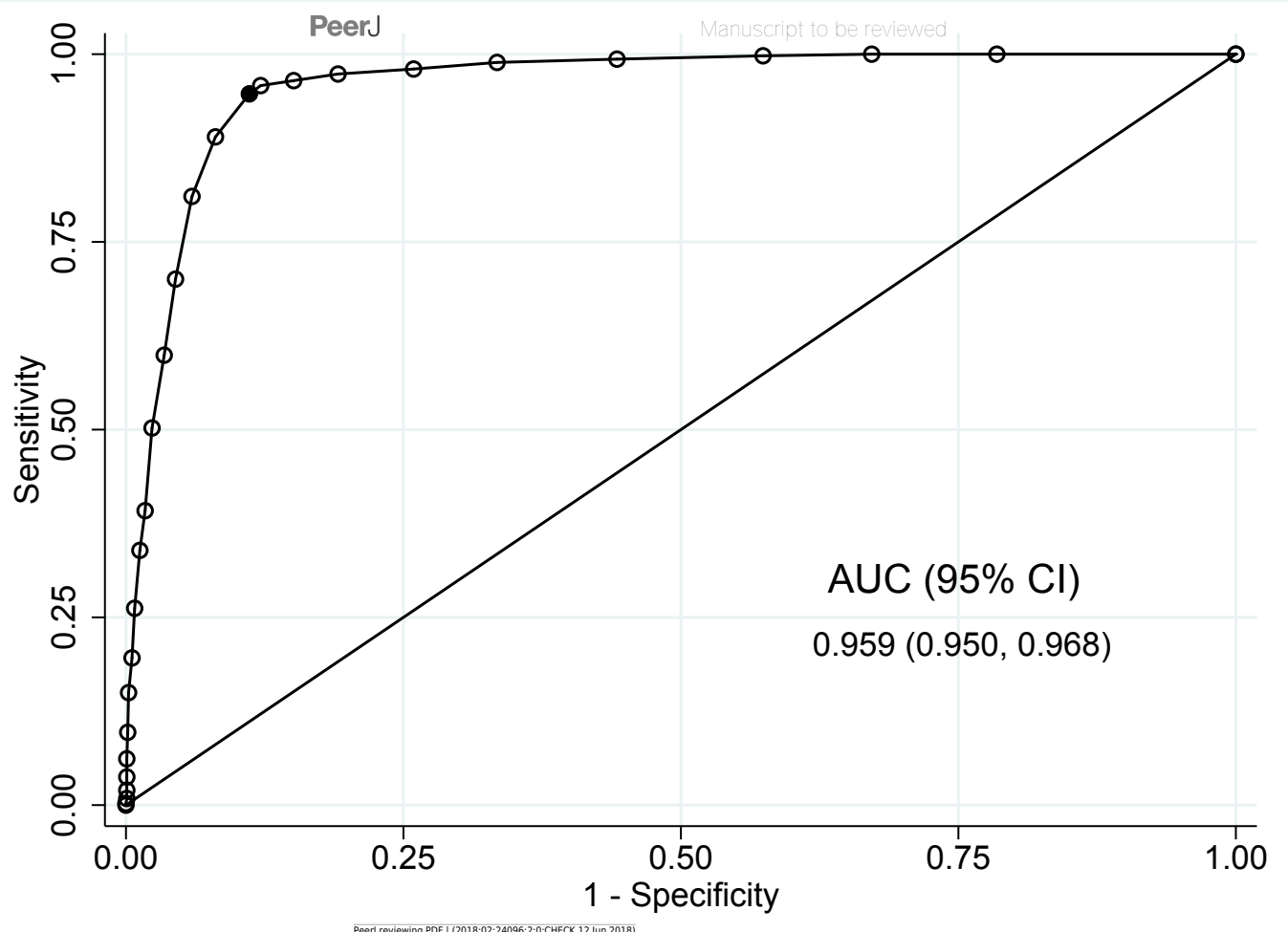

